\title{
A SÍNTESE DOS SESQUITERPENOS BAQUENOLIDAS
}

\author{
Timothy John Brocksom* e Ursula Brocksom \\ Departamento de Química, Universidade Federal de São Carlos, CP 676, 13560-970 São Carlos - SP, Brasil \\ Maurício G. Constantino \\ Departamento de Química, Faculdade de Filosofia, Ciências e Letras de Ribeirão Preto, Universidade de São Paulo, $14040-901$ \\ Ribeirão Preto - SP, Brasil
}

Recebido em 12/1/08, aceito em 9/4/08, publicado na web em 29/4/08

\begin{abstract}
THE SYNTHESIS OF THE BAKKENOLIDE SESQUITERPENES. The bakkenolide group of sesquiterpenes has been a focal point of synthetic efforts amongst several research laboratories all linked to our common origins at the Chemistry Institute at the State University in São Paulo. In this review we would like to present a historical perspective of this research, with unpublished background information, and a definitive retrosynthetic analysis of these molecules. This chemistry spans the time period from 1972 to 2006 , and traces the initial steps with Prof. Dr. Helena Ferraz to the present day.
\end{abstract}

Keywords: total synthesis; sesquiterpenes; bakkenolides.

\section{INTRODUÇÃO: SÍNTESE ORGÂNICA DE PRODUTOS NATURAIS}

A síntese total de produtos naturais sempre foi uma atividade relevante entre os químicos orgânicos, desde o século retrasado e a tentativa de Perkin de transformar alil-anilina em quinina. ${ }^{1}$ Os motivos deste interesse incluem a necessidade de confirmar as estruturas propostas, antes do advento dos métodos espectroscópicos e difração de raio-X, o que ainda continua hoje nos casos esporádi$\cos$ de equívocos. ${ }^{2}$ A transformação de moléculas simples e disponíveis em produtos de maior valor agregado sempre foi relevante na indústria, e hoje encontra o seu ponto alto na indústria farmacêutica. ${ }^{3}$ Após a $2^{\text {a }}$ Guerra Mundial, as pesquisas de Robert B. Woodward elevaram o nível de qualidade a uma arte consagrada, ${ }^{4}$ o que permitiu a introdução de importantes conceitos em Química Orgânica, inclusive as regras de Woodward-Hoffmann. ${ }^{5}$ Depois, Elias J. Corey formulou a disciplina acadêmica de síntese orgânica, através de análise retrossintética. ${ }^{6}$ Atualmente, a pesquisa acadêmica em síntese orgânica se justifica plenamente pelo treinamento oferecido aos pós-graduandos e pós-doutorandos, que almejam ingressar na indústria farmacêutica multinacional. ${ }^{7}$ Por fim, e provavelmente mais importante ainda para o pesquisador, há a imensa satisfação intelectual em planejar e depois executar uma síntese de uma molécula complexa. ${ }^{8}$ No aspecto geral do avanço da química orgânica, há também a questão de testar os limites do conhecimento, verificar as idéias, os conceitos e as teorias atuais. ${ }^{9}$

Partindo deste ponto, o químico orgânico de síntese procura as suas moléculas alvos dentro da imensidão dos produtos naturais secundários conhecidos, e neste artigo queremos fixar a atenção em um tipo de sesquiterpeno que passou a se chamar de baquenolida. ${ }^{10}$ A primeira pergunta do leigo sempre é porque esta molécula, e poderíamos justificar nossa escolha pelos argumentos clássicos de estrutura desafiante, atividades biológicas relevantes e neste caso ainda o fato que as baquenolidas têm uma origem biossíntética "não isoprenóide", o que sugere confirmação da sua estrutura por síntese. No entanto, a verdade é um pouco mais complexa, nem sempre tão acadêmica, e provavelmente muito mais freqüentemente envolve relações pessoais e momentos em tempo, como veremos a seguir.

\section{A ORIGEM DO PROJETO BAQUENOLIDA}

No final da década de 60, o Prof. Dr. William S. Johnson da Universidade de Stanford, Califórnia, nos Estados Unidos, preparava a sua disciplina tradicional de pós-graduação, Chemistry 225, que versava sobre a síntese de moléculas orgânicas relevantes de interesse sintético ou de produtos naturais recém descobertos. No início de 1968 Abe e colaboradores descreveram ${ }^{11}$ um novo sesquiterpeno ${ }^{12}$ isolado de uma planta japonesa de nome local Bakke, que originou o nome da substância e, depois, de outras estruturalmente relacionadas. Simultaneamente Naya e colaboradores ${ }^{13}$ isolaram fukinanolide, de uma planta japonesa de nome local Fuki. De fato os dois produtos naturais possuem a mesma estrutura e por consenso mais recente utiliza-se atualmente o nome geral "baquenolidas". Como Abe e colaboradores publicaram os seus resultados numa revista de maior prestígio, ficaram com a fama e o nome do composto BaquenolidaA, e foi assim que o Prof. Johnson se interessou pela molécula e incluiu na sua disciplina daquele ano. Os alunos da disciplina Chemistry 225 tinham a obrigação de preparar propostas de síntese para cada molécula em forma de seqüências de reações, com reagentes, condições, estimativas de rendimento para cada etapa, e referências bibliográficas de cada reação proposta.

O Dr. Simon Campbell, até então pós-doutorando no laboratório do Prof. Johnson, aceitou participar no programa NAS/CNPq ${ }^{14}$ no Instituto de Química da Universidade de São Paulo, e trouxe o problema de síntese total da Baquenolida-A, vindo justamente das aulas e seminários do Prof. Johnson.

A seguir apresentamos a estrutura convencional e tri-dimensional desta molécula (Figura 1), e a sua relação biossintética provável com

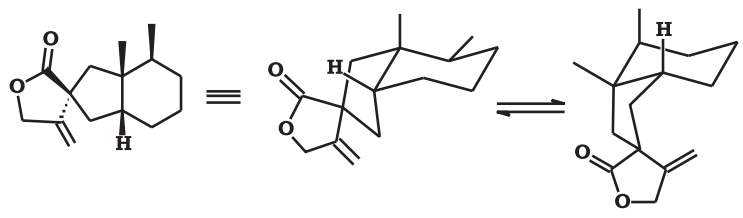

Figura 1. Baquenolida-A

*e-mail: brocksom@terra.com.br 
os sesquiterpenos do tipo eremofilano, ${ }^{15}$ eudesmano, germacrano e finalmente com o pirofosfato de farnesila (Esquema 1).
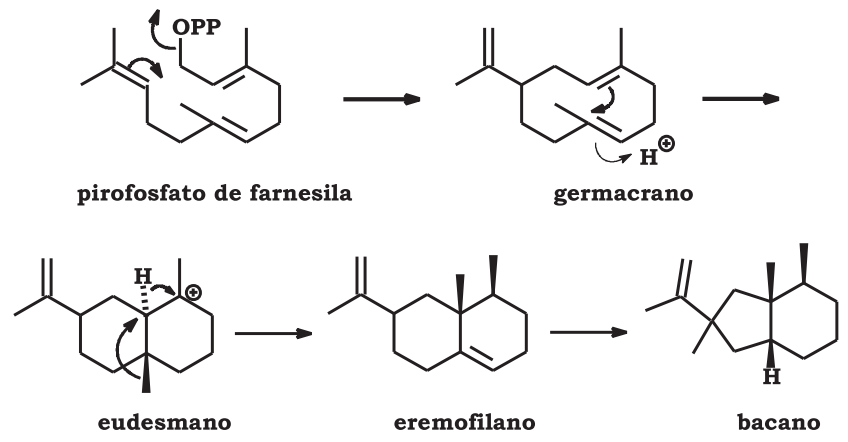

Esquema 1

Evidentemente, não nos compete discutir sobre os motivos que levaram o Prof. Johnson a escolher Baquenolida-A (deste ponto em diante denominada simplesmente B-A) como objeto de síntese em 1968 numa disciplina acadêmica, e nem porque o Dr. Campbell elegeu esta molécula para trazer ao Brasil, e dar início a uma linha de pesquisa de longa data e com tantos resultados importantes. Podemos sim dar a nossa opinião sobre o produto natural B-A, e o que o tornava relevante no final da década de 60 e continua oferecendo bastante interesse até hoje.

\section{ARQUITETURA MOLECULAR DA BAQUENOLIDA-A}

A primeira atividade intelectual do pesquisador em planejar uma síntese total de um produto natural é examinar atentamente a estrutura tri-dimensional da molécula alvo. No caso de B-A, podemos verificar a presença de um sistema cis-hidrindano, ou seja, um anel de seis carbonos em fusão cis com um anel de cinco carbonos, um grupo metílico na junção dos anéis e um segundo grupo metílico no carbono vizinho, também com uma relação cis entre estes substituintes. Ademais, no anel de cinco carbonos há em espiro, um anel butirolactônico com um grupo exo-metileno em $\beta$. A Figura 1 apresenta as duas conformações mais prováveis desta molécula, destacando os quatro centros estereogênicos, o átomo de carbono quaternário em espiro, e especialmente a unidade espiro-lactônica presente somente neste pequeno grupo de produtos naturais.

Os desafios sintéticos podem ser enumerados, lembrando que alguns eram realmente inéditos e, portanto, ainda sem solução. Primeiro, há a unidade de um cis-hidrindano com dois grupos metílicos em carbonos vizinhos, e com funcionalidade no carbono central do anel de cinco carbonos. Partindo deste mesmo átomo de carbono, destacando mais uma vez que este carbono é quaternário, há a unidade espiro-lactônica $\beta$-metilênica. O próximo passo no planejamento é fazer análise retrossintética ${ }^{6}$ levando em conta a arquitetura molecular e os desafios individuais sintéticos. Neste momento do planejamento, há uma reflexão sobre a necessidade e a conveniência de investir esforços em estudos modelos de unidades menores da molécula, para depois juntar estes estudos numa síntese total. $\mathrm{Na}$ época era comum praticar esta abordagem, mas acreditamos que atualmente caiu em desuso, até porque houve muitos casos em que os modelos eram sintetizados com sucesso, mas ao juntar os processos não ocorria correspondência de resultado na molécula alvo completa.

\section{ANÁLISE RETROSSINTÉTICA DA BAQUENOLIDA-A E OS MODELOS}

É muito simples dividir a molécula de B-A (1) em duas partes, envolvendo por um lado uma $\beta$-metileno-espiro-butirolactona ligada a um anel de cinco carbonos 2 , e por outro lado um cishidrindano bis-metilado 3. O Esquema 2 apresenta esta proposta de modelos em forma de análise retrossintética.

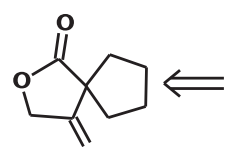

2

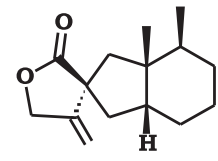

B - A (1)

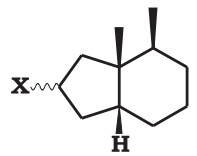

3
Esquema 2. $X$ = funcionalidade

Os primeiros estudos visavam a molécula 2 , a $\beta$-metileno- $\gamma$ butirolactona espiro ciclopentano funcionalizada no carbono central. Nesta homenagem à Profa. Dra. Helena Ferraz, pretendemos destacar a sua participação fundamental no desenvolvimento dos estudos sintéticos para as baque-nolidas, e neste sentido citamos a excelente revisão sobre as sínteses totais, publicada por seu exorientado Prof. Dr. Luiz Fernando Silva Jr. ${ }^{16}$

Em 1977 foram publicados os primeiros trabalhos da Profa. Helena envolvendo justamente a molécula modelo 2, que são apresentados no Esquema 3. ${ }^{17-19}$ Optamos por não apresentar as condições reacionais e os rendimentos das reações das sínteses para não detalhar de forma excessiva e desnecessária e, ainda, estes dados constam nas publicações citadas.

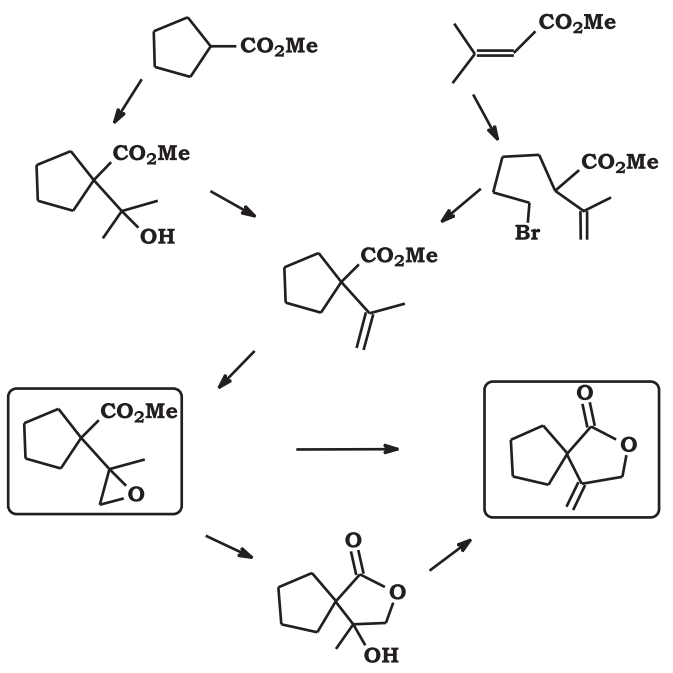

Esquema 3

Simultaneamente iniciamos estudos visando à síntese do modelo 3, o cis-hidrindano bis-metilado, e as nossas primeiras idéias focalizaram a reação de Diels-Alder ${ }^{20}$ como reação chave para a construção do anel de seis carbonos. Isto porque a junção de dois anéis em fusão cis sugere fortemente uma cicloadição de acordo com as regras Woodward-Hoffmann. O estudo da cicloadiçaõ [4+2] entre o cis-piperileno e o anidrido citracônico não levou a resultados significativos para a síntese da B-A, porém o estudo expandido da reação incluindo também o trans-piperileno e dienófilos de anidrido maleico, conduziu a uma publicação ${ }^{21}$ tão relevante que foi utilizada como exercício em livro texto importante de pós-graduação. ${ }^{22}$ Os resultados completos (Esquema 4) deste estudo foram apresentados em revista nacional, ${ }^{23}$ onde também foram destacados os primeiros estudos sobre a versão intramolecular da reação de Diels-Alder (IMDA) e que são relevantes para algumas sínteses bem mais recentes discutidas a seguir. 
<smiles>[R]C1=CC(=O)OC1=O</smiles><smiles>[R]C1=CC(=O)OC1=O</smiles><smiles>[Y]C/C=C\C=C/[C@H](O[R20])/C(C)=C/C=C</smiles>

Esquema 4. $R=$ Me ou $H ; X=Y=$ funcionalidade

\section{ANÁLISE RETROSSINTÉTICA DA BAQUENOLIDA E ALGUMAS SÍNTESES TOTAIS}

Como os resultados não foram muito positivos na direção de uma síntese do cis-hidrindano bis-metilado 3 utilizando a reação de Diels-Alder, houve um certo recuo, quando aconteceu o próximo encontro fundamental para o futuro sucesso. Na terceira Escola de Verão em Química Orgânica ${ }^{24}$ na UFSCar, em 1983 o Prof. Dr. Andrew E. Greene, da Université de Grenoble, França, ofereceu uma disciplina sobre aspectos de síntese incluindo trabalhos de seu próprio laboratório. Certamente, destacou a importância das cicloadições [2+2] entre dicloroceteno e alcenos, e a posterior expansão de anel, que conduz efetivamente a uma metodologia de pentanelação.

Uma análise retrossintética expandida desta possibilidade para a síntese de $\mathbf{3}$ inclui as alternativas extremamente relevantes que são expansão de anel de um sistema biciclo[4.2.0]octano apropriado, e a contração do anel de seis carbonos de uma decalina apropriada. Esta discussão simplesmente destaca a dificuldade de sintetizar anéis ciclopentânicos de forma direta, o que tem a ver com o seu número impar de carbonos. O Esquema 5 apresenta estas duas opções, junto com as de Diels-Alder nas versões intermolecular e intramolecular. Completando o Esquema 5, introduzimos uma gama muito grande de desconexões de duas ligações carbono-carbono, com um substrato propício para formar o produto com os dois grupos metílicos em relação cis. Esta proposta implica numa reação do tipo tandem-dominó, onde estas duas ligações serão formadas, e os dois anéis fechados simultaneamente.
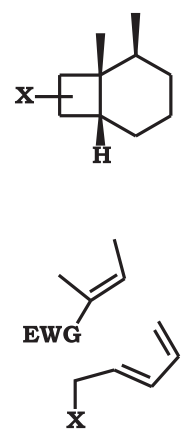
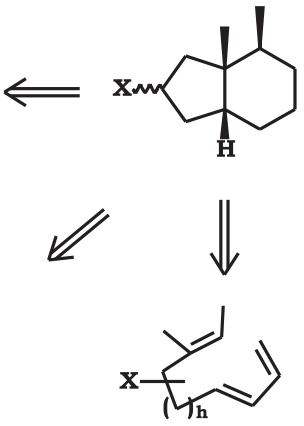
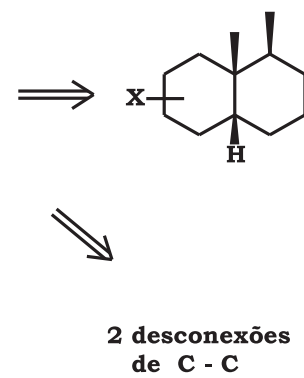
de $\mathbf{C}-\mathbf{C}$
Esquema 5. EWG = grupo retirador de densidade eletrônica; $X=$ funcionalidade

A partir desta nova análise retrossintética, e as perspectivas de incorporar a química do Prof. Greene, foi estabelecido um projeto conjunto. É relevante comentar aqui que a proposta avança no sentido que inverte a questão de anelação. Nas propostas de cicloadição [4+2] haveria a formação do anel de seis carbonos em cima de um substrato de cinco átomos, enquanto a nova proposta de cicloadição [2+2] cria o anel de quatro (e depois cinco) membros em cima de um substrato de seis carbonos. Obviamente substratos de seis átomos de carbono (cicloexanos) são muito mais disponíveis de que substratos de cinco átomos.

Neste projeto conjunto UFSCar-Grenoble ainda contamos com a participação do Prof. Dr. Fernando Coelho, naquele momento pós-graduando no laboratório do Prof. Greene. Assim o planejamento sintético foi transformado em sucesso pela competência experimental e dedicação do Prof. Coelho, e publicamos ${ }^{25}$ a primeira de uma longa lista de sínteses determinadas pela análise retrossintética do Esquema 5. O Esquema 6 apresenta a síntese enantiosseletiva da B-A a partir do 2,3-dimetil-cicloexeno enantiopuro, como exemplo da execução da nossa análise retrossintética.

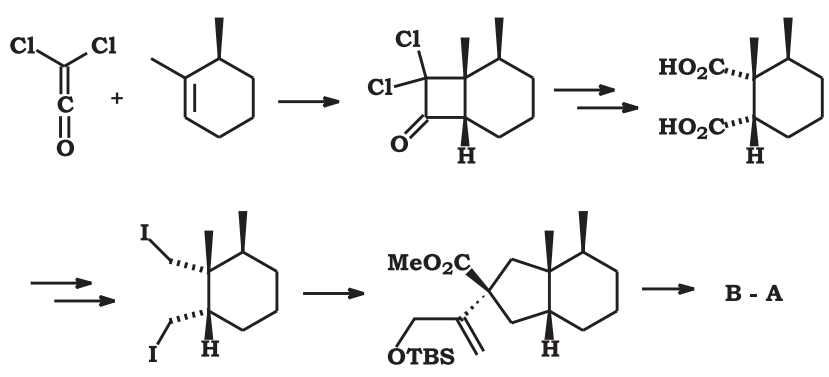

Esquema 6

A historia completa ${ }^{26}$ destas sínteses baseadas na abordagem planejada numa Escola de Verão na UFSCar, inclui sínteses de baquenolidas poli-funcionalizadas e nas suas formas enantiomericamente puras. É interessante comentar que esta última publicação, contando o desenvolvimento deste projeto ao longo de duas décadas, envolve a participação de quatro "brasileiros" (T. J. Brocksom, F. Coelho, M. E. F. de Lima e A. M. Kanazawa).

Voltando às análises retrossintéticas já apresentadas, podemos verificar que ainda há várias opções interessantes que deveriam ser investigadas, e as discutiremos a seguir.

\section{ANÁLISE RETROSSINTÉTICA DA BAQUENOLIDA E ESTU-DOS RELEVANTES}

Como havia sido previsto e experimentado ${ }^{23}$ (Esquema 4), há uma grande possibilidade de executar uma reação de Diels-Alder intramolecular (IMDA). ${ }^{20}$ Assim voltamos a esta possibilidade, mas com a vantagem da experiência acumulada e muitas novidades a serem experimentadas. Nesta breve homenagem não será possível explanar sobre tudo o que foi realmente executado no laboratório, mas o Esquema 7 fornece uma visão global dos nossos esforços. ${ }^{27}$ As unidades do dienófilo derivado de ácido tíglico, e dienos de cinco e seis carbonos foram sintetizadas, e ligadas nas três modalidades previstas para reações IMDA: ligação covalente, onde os átomos X e Y farão parte do produto; ligação covalente, onde $\mathrm{X}$ e Y são grupos de ligação, porém não farão parte do produto final (simplesmente espaçadores) e, ligação temporária através de complexação com metais.

Em resumo, não houve evidências claras da formação de intermediários avançados para a síntese das baquenolidas, e acreditamos que as moléculas substratos não aceitam adotar conformações adequadas para a reação de IMDA. Ou seja, as energias de 


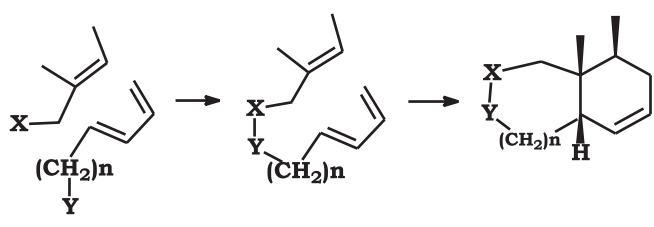

Esquema 7. $n=1$ ou $2 ; X=Y=$ funcionalidade

ativação para as conformações ideais são maiores de que para outras reações paralelas ou são inacessíveis.

Uma proposta relevante do Esquema 5 é partir de um substrato decalínico e efetuar uma contração de anel para gerar um hidrindano. A grande vantagem desta abordagem seria na maior disponibilidade de substratos decalínicos.

Esta idéia foi posta em prática pelo Prof. Dr. Gil Valdo José da Silva, durante o seu doutorado sob a orientação do Prof. Dr. Nicola Petragnani na década de $80,{ }^{28}$ como pode ser visto no Esquema 8.
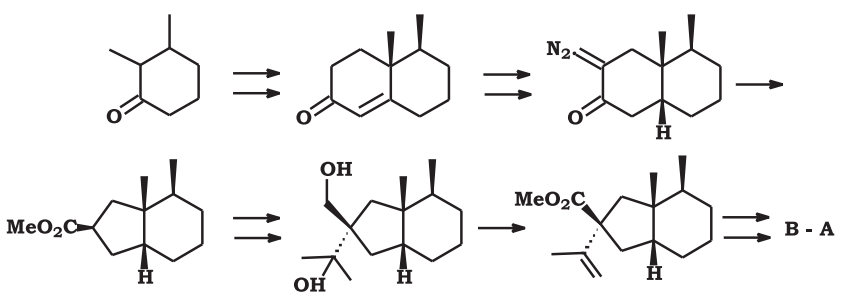

Esquema 8

A Profa. Helena havia se tornado uma especialista na química orgânica de tálio, e o uso deste na contração de anéis. Assim, foi feito um esforço neste sentido, sem no entanto alcançar o objetivo principal de sintetizar uma baquenolida, ${ }^{29,30}$ por contração de uma octalona cis-dimetilada. O Esquema 9 procura retratar os resultados principais deste estudo durante o doutorado do Dr. Tiago de Oliveira Vieira.

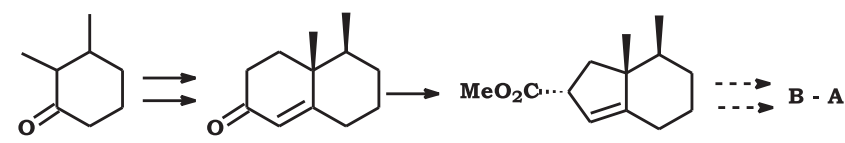

Esquema 9

\section{UMA SÍNTESE TOTAL SOMANDO OS MODELOS}

Finalmente, aproveitando nossa recente experiência com utilização de $\mathrm{NbCl}_{5}$ para catalisar reações de cicloadição, a síntese totalmente nacional envolvendo a reação de Diels-Alder para gerar a unidade do tipo 3 e a aplicação dos modelos para anelar o sistema $\beta$-metileno-espiro-butirolactona, foi realizada em $2006^{31,32}$ pelo Dr. Kleber Thiago de Oliveira. Esta síntese total está apresentada no Esquema 10.

O cis-dimetil-cis-cicloexano substituído foi sintetizado logo no início da síntese, e as funções ajustadas para incorporar a espiroanelação da butirolactona $\beta$-metileno e, assim, terminar a síntese da B-A.

\section{CONCLUSÃO E PERSPECTIVAS}

Desde a publicação ${ }^{16}$ em 2001 da revisão sobre as sínteses das baquenolidas, houve ainda bastante esforço em outros laboratórios para sintetizar as baquenolidas, e devemos atualizar esta bibliografia citando algumas publicações mais relevantes. O grupo de Srikrishna, ${ }^{33}$ como de costume inicia a síntese a partir da carvona

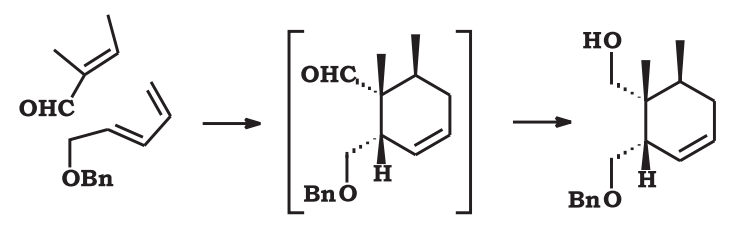<smiles>C=C(C)C(C[C@H]1CCC[C@H](C)[C@]1(C)CI)C(=O)OCCC</smiles>

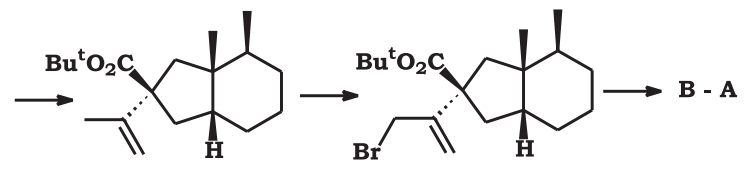

Esquema 10

natural e aproveita a sua quiralidade e funcionalidade, porém a síntese leva ao epímero da B-A através de uma ciclização radicalar. Reddy ${ }^{34}$ utiliza uma estratégia interessante envolvendo uma reação de Diels-Alder intermolecular em tandem com uma reação de aldol para gerar o sistema hidrindano. O grupo de Greene ${ }^{35}$ continua explorando variações sobre a cicloadição [2+2], enquanto o grupo de Back $^{36}$ também se interessou pela aplicação da reação de Diels-Alder intramolecular para gerar o sistema completo da B-A. Neste último trabalho a ciclização da reação IMDA é conduzida em condições extremamente vigorosas e leva a uma mistura diaestereomérica muito complexa.

Em conclusão, podemos constatar que uma molécula relativamente simples pelos padrões de Nicolaou, ${ }^{37}$ um mero sesquiterpeno com quinze carbonos e somente quatro centro estereogênicos, uma única função orgânica (a lactona), deu tanto trabalho intelectual e de bancada para tanta gente. Até o Prof. David A. Evans ${ }^{38}$ deu seus primeiros passos numa síntese de B-A. E por quais razões? Tentamos mostrar um pouco destas razões, que nem sempre refletem uma procura puramente científica, mas uma seqüência de acontecimentos fortuitos e de relações pessoais, o que reforça uma visão de que a ciência da Síntese Orgânica é eminentemente uma atividade exercida por pessoas.

As diversas sínteses acadêmicas de B-A já realizadas certamente não esgotam o assunto; novas sínteses serão, com certeza, desenvolvidas, na busca da melhor, da definitiva, da mais elegante, da mais eficiente, da mais inovadora, da mais... Certamente há o que melhorar nas sínteses publicadas, seja a redução do número de etapas, seja diminuir a linearidade e aumentar a convergência, seja melhorar o rendimento de algumas reações chaves (e nem tão chaves), seja (talvez principalmente) aumentar a estereosseletividade (nem sempre muito alta) na geração dos três centros estereogênicos contíguos da junção dos anéis do sistema hidrindano e do carbono quaternário espiro.

As sínteses publicadas até hoje demonstram algumas verdades sobre a área, em que não há nunca uma síntese perfeita ou ideal, ${ }^{39}$ mas, sempre uma abordagem própria de um grupo de pesquisa; em conjunto, as várias sínteses levam a uma avaliação relevante sobre o desenvolvimento da química orgânica. É interessante constatar que as sínteses publicadas da molécula B-A começaram em 1973 e vão até 2006 (por enquanto), envolvendo aproximadamente 10 grupos em vários países, e com grande independência entre estes. A história da síntese orgânica mostra que apenas algumas poucas moléculas permanecem efetivamente por muito tempo excitando a nossa imaginação, e ser- 
vindo como modelos para demonstrar a eficácia de metodologias ou de estratégia novas. As baquenolidas fazem parte deste seleto grupo!

\section{AGRADECIMENTOS}

Num empreendimento científico desta natureza, houve a participação efetiva de mais pessoas de que poderiam ser citadas no texto, mas seus nomes aparecem nas referências bibliográficas. Certamente, devemos agradecer ao programa NAS/CNPq por ter dado o pontapé inicial, e um apoio financeiro que, no entanto, pode ser considerado muito pequeno pelos padrões atuais. Posteriormente, às agências de fomento FAPESP, CNPq e CAPES pelas bolsas de estudos e pelos auxílios.

Ao Instituto de Química da USP em São Paulo por ter oferecido abrigo fundamental no início deste projeto, e depois por ter deixado ir embora. Ao Prof Nicola Petragnani que nem sempre entendeu, mas sempre apoiou e ajudou, e sem o qual a Profa. Helena não teria começado a aprender tudo. Finalmente, à Profa. Helena Ferraz pelo convívio científico e humano durante estas três décadas, que para nós que aqui permanecemos, ficará para sempre como uma lição de amor à vida.

\section{REFERÊNCIAS E NOTAS}

1. Fleming, I.; Selected Organic Syntheses, John Wiley and Sons: London, 1973; veja capítulo 2.

2. Nicolaou, K. C.; Snyder, S. A.; Angew. Chem., Int. Ed. 2005, 44, 1012.

3. A síntese do fármaco para o tratamento de AIDS indinavir (Crixivan ${ }^{\circledR}$ ) pela indústria Merck é considerada uma marca de complexidade; veja Pilli, R. A.; Química Nova na Escola 2001, n. 14, 16.

4. Robert B. Woodward (Prêmio Nobel em Química, 1965) é considerado por quase todos como o grande mestre da arte de planejar e executar síntese total de produtos naturais; veja, por exemplo, em Smith, M. B.; Organic Synthesis, McGraw-Hill, $2^{\text {nd }}$ ed., 2002, cap. 10, em especial p. 818.

5. Woodward, R. B.; Hoffmann, R.; The Conservation of Orbital Symmetry, Verlag Chemie-Academic Press: Weinheim, 1970.

6. Corey, E. J.; Cheng, X-M.; The Logic of Chemical Synthesis, John Wiley and Sons: New York, 1989; Prêmio Nobel em Química, 1990.

7. O desenvolvimento de novos fármacos exige a ativa participação de químicos orgânicos sintéticos, de tal forma que a indústria farmacêutica financia a pesquisa e pós-graduação em grupos importantes e em países do primeiro mundo. Enquanto isto, aqui no Brasil não há ainda esta percepção da importância.

8. A disciplina acadêmica de Síntese Orgânica exige e permite exercitar todos os conhecimentos atuais em química, e interage com todos os avanços em identificação estrutural e separação analítica, os conceitos fundamentais mecanísticos de físico-química, e utiliza constantemente reagentes inorgânicos e organometáli-cos.

9. Veja Correia, C. R. D.; Síntese Estereosseletiva de Alcalóides e NHeterocíclos, EdUFSCar, 2001, cap. 2, para uma excelente apresentação, em língua portuguesa, dos fundamentos de síntese orgânica moderna.

10. Parece que há um consenso, entre os grupos atuantes em síntese no Brasil, de que a palavra bakkenolide é melhor traduzida como "baquenolida". A palavra em inglês junta o nome do esqueleto carbônico do tipo de sesquiterpeno bakkane, que vem da espécie de planta em que se encontra (bakke), e a terminação - olide que significa uma lactona de cinco membros.

11. Abe, N.; Onoda, R.; Shirahata, K.; Kato, T.; Woods, M. C.; Kitahara, Y.; Tetrahedron Lett. 1968, 369.

12. Um sesquiterpeno é um produto natural derivado biossinteticamente de pirofosfato de farnesila, portanto conta com quinze carbonos no esqueleto carbônico principal.
13. Naya, K.; Takagi, I.; Hayashi, M.; Nakamura, S.; Kobayashi, M.; Katsumura, S.; Chem. Ind. 1968, 318.

14. Campbell, S. F.; Quim. Nova 2007, 30, 1393; veja também Brocksom, T. J.; Quim. Nova 2007, 30, 1394.

15. Hayashi, K.; Nakamura, H.; Mitsuhashi, H.; Chem. Pharm. Bull. 1973, 21, 2806; nesta síntese, o produto natural fukinone que é um sesquiterpeno eremofilano, foi transformado em Baquenolida-A, o que corresponde a uma síntese biomimética e, portanto, apóia a proposta biogenética.

16. Silva Jr., L. F.; Synthesis 2001, 671. Nesta ótima revisão as estruturas, a ocorrência e as atividades biológicas de diversas baquenolidas são apresentadas, tornando desnecessário repetir aqui.

17. Petragnani, N.; Brocksom, T. J.; Ferraz, H. M. C.; Constantino, M. G.; Synthesis 1977, 112.

18. Petragnani, N.; Brocksom, T. J.; Ferraz, H. M. C.; Constantino, M. G.; Quim. Nova 1978, 1, 8 .

19. Brocksom, T. J.; Constantino, M. G.; Ferraz, H. M. C.; Synth. Commun. 1977, 7, 483

20. Brocksom, T. J.; Nakamura, J.; Ferreira, M. L.; Brocksom, U.; J. Braz. Chem. Soc. 2001, 12, 597.

21. Brocksom, T. J.; Constantino, M. G.; J. Org. Chem. 1982, 47, 3450.

22. Carey, F. A.; Sundberg, R. J.; Advanced Organic Chemistry, Part A: Structure and Mechanisms, Plenum Press, $2^{\text {nd }}$ ed., 1984, p. 578 e 708 e a $5^{\text {a }}$ ed. saiu pela Springer, 2007, p. 958 e 1166.

23. Brocksom, T. J.; Constantino, M. G.; An. Acad. Brasil. Ciênc. 1982, 54, 655 .

24. As Escolas de Verão em Química Orgânica da UFSCar são uma continuação das Escolas de Julho em Química Orgânica organizadas pelo eminente Prof. Dr. Otto R. Gottleib, na Universidade Federal Rural do Rio de Janeiro. Dr. Otto permitiu o deslocamento de sua idéia brilhante, da UFRRJ para a UFSCar no final da década de 70. No início da década de 80, o coordenador do ano, o Prof. Dr. Eliezer J. L. Barreiro, durante a sua curta, porém marcante permanência na UFSCar, re-iniciou a participação internacional das Escolas e indicou o Prof. Andrew E. Greene como ministrante de uma disciplina.

25. Greene, A. E.; Deprés, J-P.; Coelho, F.; Brocksom T. J.; J. Org. Chem. 1985, $50,3943$.

26. Brocksom, T. J.; Coelho, F.; Deprés, J-P.; Greene, A. E.; de Lima, M. E. F.; Hamelin, O.; Hartmann, B.; Kanazawa, A. M.; Wang, Y.; J. Am. Chem. Soc. 2002, 124, 15313.

27. Nakamura, J.; Tese de Doutorado, Universidade Federal de São Carlos, Brasil, 2003: Nakamura, J.; Brocksom, U.; Brocksom, T. J.; Resumos do 9th Brazilian Meeting on Organic Synthesis, Curitiba, Brasil, 2001.

28. Da Silva, G. V. J.; Tese de Doutorado, Universidade de São Paulo, Brasil, 1984.

29. Vieira, T. de O.; Tese de Doutorado, Universidade de São Paulo, Brasil, 2005.

30. Ferraz, H. M. C.; Vieira, T. de O.; Silva Jr. L. F.; Synthesis 2006, 2748.

31. Constantino, M. G.; de Oliveira, K. T.; Polo, E. C.; da Silva, G. V. J.; Brocksom, T. J.; J. Org. Chem. 2006, 71, 9880.

32. De Oliveira, K. T.; Tese de Doutorado, Universidade de São Paulo, Brasil, 2006.

33. Srikrishna, A.; Reddy, T. J.; ARKIVOC (Gainesville, FL, U. S.) 2001, 19: Tetrahedron: Asymmetry 2003, 14, 2975.

34. Reddy, D. S.; Org. Lett. 2004, 6, 3345.

35. Hamelin, O.; Wang, Y.; Deprés, J-P.; Greene, A. E.; Angew. Chem., Int. Ed. 2000, 39, 4314.

36. Back, T. G.; Nava-Salgado, V. O.; Payne, J. E.; J. Org. Chem. 2001, 66, 4361.

37. Nicolaou, K. C.; Vourloumis, D.; Winssinger, N.; Baran, P. S.; Angew. Chem., Int. Ed. 2000, 39, 44.

38. Evans, D. A.; Sims, C. L.; Tetrahedron Lett. 1973, 4691; Evans, D. A.; Sims, C. L.; Andrews, G. C.; J. Am. Chem. Soc. 1977, 99, 5453.

39. Wender, P. A.; Miller, B. L. Em Organic Synthesis: Theory and Applications; Hudlicky, T., ed.; Elsevier/JAI Press, 1993, vol. 2, p. 26: Wender, P. A.; Handy, S. T.; Wright, D. L.; Chem. Ind. 1997, 765; veja também, Wender, P. A.; Chem. Rev. 1996, 96, 1. 